\title{
Iterative methods for thermographic reconstruction using the virtual wave concept
}

\author{
by P. Burgholzer*, G. Thummerer**, G. Mayr ${ }^{\star *}$, P. Kovacs ${ }^{\star * *}$, M. Haltmeier**** \\ * RECENDT, Research Center for Non-Destructive Testing, Linz, Austria \\ ** University of Applied Sciences Upper Austria, Wels, Austria \\ *** Institute of Signal Processing, Johannes Kepler University and Silicon Austria Labs (SAL), Linz, Austria \\ **** Department of Mathematics, University of Innsbruck, Innsbruck, Austria
}

\begin{abstract}
The achievable spatial resolution in thermography decreases with the imaging depth, which leads to blurred images for deeper lying structures. The resolution limit has its origin in the second law of thermodynamics: heat diffusion on its way from the imaged structure to the sample surface leads to entropy production that is equal to the loss of information. In order to address this issue, additional available information about the sample has to be included in the regularization of the inverse problem, such as positivity or sparsity of the signals. We combine the benefits of acoustic and thermal imaging using non-linear reconstruction algorithms combined with the recently introduced virtual wave conceopt.
\end{abstract}

\section{Introduction}

A new approach for thermographic imaging, the so-called virtual wave concept (VWC) improves the quality of the $2 \mathrm{D}$ or $3 \mathrm{D}$ thermographic image reconstruction by partly compensating the diffusion related information loss [1]. The idea of VWC is to transform the surface temperature signal, detected by an infrared (IR) camera, into an acoustic virtual wave field [2]. The first transformations of diffusive electromagnetic waves to acoustic wave fields have been shown by Lee et al. [2] and Gershenson [3] for geophysical applications. While the surface temperature fulfils the diffusion equation, the virtual wave field obeys the wave equation. In contrast to the diffusion equation, the wave equation describes a reversible process. However, reconstruction methods, well known from ultrasonic testing can be employed for 3D thermographic imaging [4]. The VWC is a two-step inverse process. The first inverse problem is severely ill-posed due to information loss that is equal to the entropy production during heat diffusion. To enhance the quality of the inverse solution we make use of additional information in form of positivity and sparsity [5]. The one-dimensional wave has a non-negative solution for all times. Hence, the additional information positivity is direct applicable [6] for reconstructing the virtual wave field. For a multidimensional problem, this is not directly true. However, we can calculate the circular projections for the 2D case and the spherical projections for the 3D case, to obtain positivity. The spherical projections correspond to a time integral of the $3 \mathrm{D}$ virtual wave. To calculate the circular projections in 2D, the Abel transformation can be applied [7]. Sparsity is introduced by an appropriate formulation of the objective function.

\section{The virtual wave concept}

The formal relationship between temperature field $T(\mathbf{r}, t)$ and virtual wave field $T_{\mathrm{virt}}\left(\mathbf{r}, t^{\prime}\right)$, for the same position vector $\mathbf{r}$ but different time scales $t$ and $t^{\prime}$, is given by a Fredholm integral of the first kind:

$$
T(\mathbf{r}, t)=\int_{-\infty}^{\infty} K\left(t, t^{\prime}\right) T_{\text {virt }}\left(\mathbf{r}, t^{\prime}\right) \mathrm{d} t^{\prime} \text { with } K\left(t, t^{\prime}\right)=\frac{c}{\sqrt{\pi \alpha t}} e^{-\frac{c^{2}\left(t^{\prime}\right)^{2}}{4 \alpha t}} \text { for } t>0
$$

The thermal diffusivity $\alpha$ and the virtual speed of sound $c$ are the characteristic parameters for heat and virtual wave propagation. While $T(\mathbf{r}, t)$ obeys the heat equation, $T_{\mathrm{virt}}\left(\mathbf{r}, t^{\prime}\right)$ fulfils the photoacoustic wave equation. The above equation is valid for a Dirac-Delta-like heating in time domain, for other temporal heating functions the solution is the convolution in time with this function. The kernel $K\left(t, t^{\prime}\right)$ works as transition function and contains the characteristic parameters $\alpha$ and $c$. It is important to note that $K\left(t, t^{\prime}\right)$ is independent of the position vector $\mathbf{r}$. That means, when considering surface temperature data, that is acquired by an IR-camera, we have a pixel-wise transformation. Since thermography data is discrete in time and space, we have to discretize the above relationship between $T(\mathbf{r}, t)$ and $T_{\text {virt }}\left(\mathbf{r}, t^{\prime}\right)$ :

$$
\mathbf{T}=\mathbf{K} \mathbf{T}_{\text {virt }}
$$

The matrix $\mathbf{K}$ has rapidly decaying singular values, hence calculating $\mathbf{T}_{\text {virt }}$ based on $\mathbf{T}$ is a severely ill-posed inverse problem. Due to this, we need regularization. In principle, we can employ direct or iterative regularization methods. By using the truncated singular value decomposition (T-SVD) or Tikhonov regularization the pseudo-inverse or MoorePenrose $\mathbf{K}^{\dagger}$ is approximated. 
In Figure 1, the process steps of the virtual wave concept based on simulated data are depicted. Here we consider an experiment, where a laser pulse heats a 2D sample to be imaged. Because of the transparency of the bulk material in the wavelength of the laser only the inclusions are heated. Consequently, they act as internal heat sources and we have a certain initial temperature distribution $T_{0}^{\text {rec }}(\mathbf{r})$. The heat diffuses to the surface, where the temporal surface temperature $T(\mathbf{r}, t)$ is recorded. Then, as mentioned before, we have to solve a severely ill-posed inverse problem. In this case, for the computation of the virtual wave field $T_{\text {virt }}(\mathbf{r}, t)$, we have employed ADMM, which is an efficient iterative algorithm for constrained optimization. We have incorporated positivity and sparsity as prior information. Sparsity is respected because we assume that we only have a few point scatterers in the sample under test and hence a sparse virtual wave field. As one can see the computed virtual wave field $T_{\text {virt }}(\mathbf{r}, t)$ meets the ideal virtual wave field $T_{\text {virt }}^{\text {ideal }}(\mathbf{r}, t)$ well but the blurring due to information loss cannot be fully eliminated. As a last step, we can apply well developed ultrasonic methods in order to reconstruct the initial temperature distribution $T_{0}^{\text {rec }}(\mathbf{r})$. Despite the rather strong blurring of $T_{\text {virt }}(\mathbf{r}, t)$ for deeper lying structures, even deeper structures can be well reconstructed by ultrasonic reconstruction in $T_{0}^{\text {rec }}(\mathbf{r})$. The ultrasound reconstruction „averages" signals from all directions and therefore the noise is reduced very effectively.

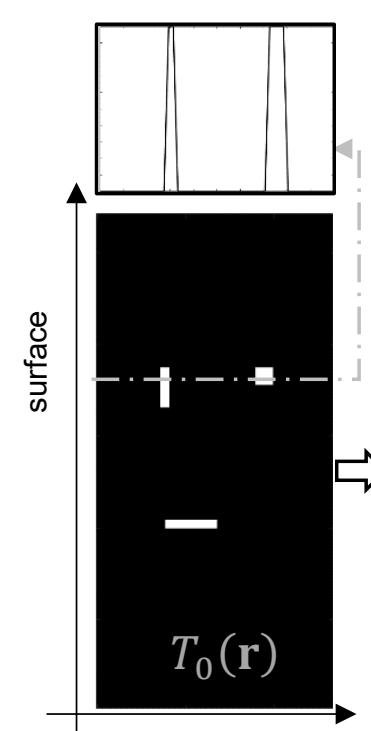

depth

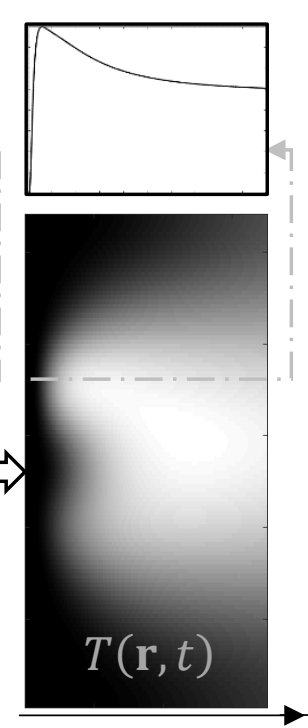

time $\mathrm{t}$
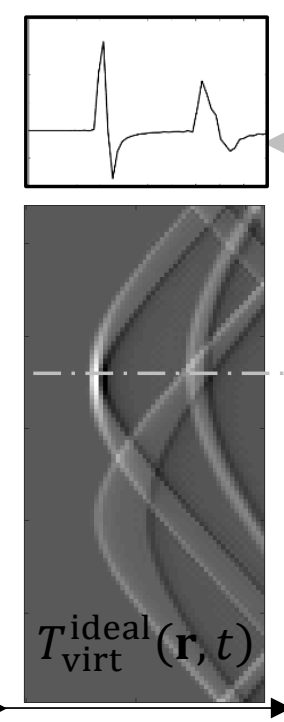

time $t$

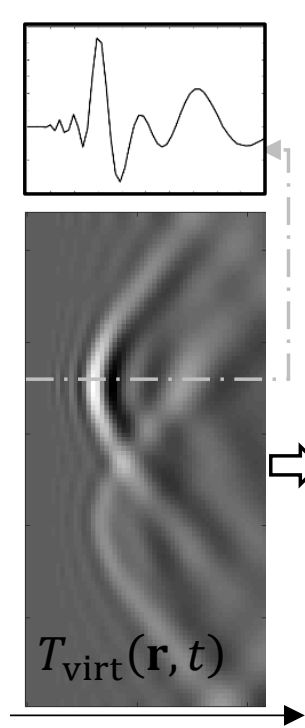

time $t$

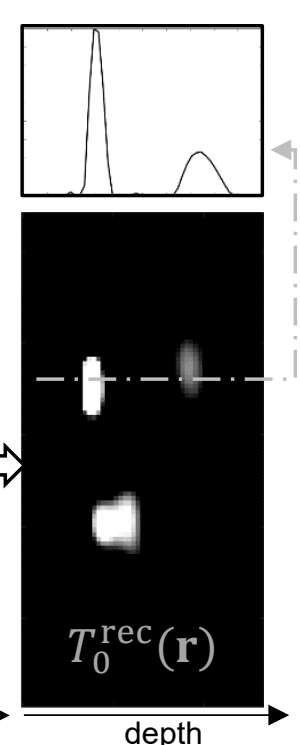

depth

Fig. 1. Process steps of the virtual wave concept: First, the internal sources are heated, by e.g. optical excitation, yielding a certain initial temperature distribution $T_{0}(\mathbf{r})$. Simultaneously, the surface temperature signal $T(\mathbf{r}, t)$ is measured using an IR-camera. Then the temperature signal is transformed into an acoustic virtual wave signal $T_{\text {virt }}(\mathbf{r}, t)$. When comparing $T_{\text {virt }}(\mathbf{r}, t)$ with the ideal virtual wave field $T_{\text {virt }}^{\text {ideal }}(\mathbf{r}, t)$, we see that the former is blurred because of entropy production during heat diffusion. As a final step, we can employ ultrasonic methods to reconstruct the initial temperature distribution $T_{0}^{\mathrm{rec}}(\mathbf{r})$.

\section{The two-step reconstruction process}

The virtual wave is an acoustic wave, which is the solution of the wave equation, and obeys the same initial conditions as the original heat diffusion problem. This transformation allows the application of ultrasound imaging algorithms such as the Synthetic Aperture Focusing Technique (SAFT). In this case, sparse regularization methods can be applied directly to the sub-problems below:

$$
\min _{\mathbf{d} \geq 0}\|\mathbf{b}-\widehat{\mathbf{K}} \mathbf{d}\|_{2}+\lambda\|\mathbf{d}\|_{1}, \quad \text { then } \quad \min _{\mathbf{v} \geq 0}\left\|\mathbf{d}-\mathbf{T}_{\mathrm{SAFT}}^{T} \mathbf{v}\right\|_{2}+\tau\|\mathbf{v}\|_{1}
$$

where $\mathbf{b}=\operatorname{vec}(\mathbf{T})$ is the vectorised form of the temporal surface temperature profile, $\mathbf{d}=\operatorname{vec}\left(\mathbf{T}_{\text {virt }}\right)$, denotes the vectorised virtual wave, $\mathbf{v}$ is the vectorized initial temperature distribution of the cross-section, and $\lambda, \tau$ are the regularization parameters. Note that we are working in the dimension of the vectorized images. Namely, if the resolution of the temporal surface temperature measurement $\mathbf{T}$ is $50 \times 50$, then $\mathbf{b} \in \mathbb{R}^{2500 \times 1}$ and $\mathbf{K}, \mathbf{T}_{\text {SAFT }}^{T} \in \mathbb{R}^{2500 \times 2500}$. Furthermore, if $\mathbf{K} \in \mathbb{R}^{50 \times 50}$ denotes the matrix (with or without Abel transform) in Eq. (2), then the corresponding block diagonal matrix $\widehat{\mathbf{K}} \in \mathbb{R}^{2500 \times 2500}$ in Eq. (3) is defined as follows: 


$$
\widehat{\mathbf{K}}=\left[\begin{array}{ccc}
\mathbf{K} & \cdots & 0 \\
\vdots & \ddots & \vdots \\
0 & \cdots & \mathbf{K}
\end{array}\right]
$$

In Eq. (3), the first sub-problem is related to thermographic imaging reconstructing the virtual wave field, while the second is an ultrasound imaging task. Note that we always got better results with the two-step reconstruction compared to one-step regularization methods using the full problem. In the proposed work, we are investigating this phenomenon in detail. Assuming that the systems of linear equations defined by $\widehat{\mathbf{K}}$ and $\mathbf{T}_{\mathrm{SAFT}}^{T}$ are independent, the two step reconstruction is more stable than solving the overall problem using the product $\widehat{\mathbf{K}} \mathbf{T}_{\mathrm{SAFT}}^{T}$. On the other hand, in thermographic imaging, the solutions are not independent, since we use the solution of the first sub-problem in the second equation. The conditionality of solving two consecutive linear inverse problems $\mathbf{b}=\mathbf{A d}$ and $\mathbf{d}=\mathbf{B v}$ can be analysed by the following identity:

$$
\operatorname{cond}(\mathbf{A B})=\|\mathbf{A B}\| \cdot\left\|\mathbf{B}^{+} \mathbf{A}^{+}\right\| \leq\|\mathbf{A}\|\|\mathbf{B}\| \cdot\left\|\mathbf{B}^{+}\right\|\left\|\mathbf{A}^{+}\right\|=\operatorname{cond}(\mathbf{A}) \cdot \operatorname{cond}(\mathbf{B}),
$$

where the first equality holds if $(\mathbf{A B})^{+}=\mathbf{B}^{+} \boldsymbol{A}^{+}$(see Theorem 3.1 in [10]). In case of $\mathbf{A}=\widehat{\mathbf{K}}$ and $\mathbf{B}=\mathbf{T}_{\mathrm{SAFT}}^{T}$, our experiments showed that Eq. (5) is satisfied for a wide range of values of the thermal diffusivity parameter $\alpha$. This implies that the condition of the one-step reconstruction is always better than or as good as the two step reconstruction. However, Eq. (5) holds only for the solutions of consecutive linear inverse problems, therefore, regularization and non-linear techniques can improve the reconstruction accuracy without increasing the numerical sensitivity. By doing so, sparsity and positivity can also be exploited in both reconstruction steps.

\section{Results}

Results for the two-stage reconstruction process using for different additional information are shown for simulations and experimentally from eddy current heated bars in an epoxy cube. For using positivity and sparsity as additional information, the Douglas Rachford splitting method [8,9] is implemented. The spatial resolution could be enhanced by a factor of two compared to linear methods, such as the truncated singular value decomposition (T-SVD) or Tikhonov regularization.

\section{REFERENCES}

[1] P. Burgholzer, M. Thor, J. Gruber and G. Mayr. "Three-dimensional thermographic imaging using a virtual wave concept.” Journal of Applied Physics, Vol. 121, No.10: p 105102-1-105102-11, 2017.

[2] K. Lee, H. G. Liu and H. F. Morrison. "A new approach to modelling the electromagnetic response of conductive media." GEOPHYSICS Vol.54, No.9: p 1180-1192, 1989.

[3] M. Gershenson. "Simple interpretation of time-domain electromagnetic sounding using similarities between wave and diffusion Propagation." Geophysics, Vol. 62, No.3: p 763-774,1997

[4] P. Burgholzer, G. Stockner and G. Mayr. "Acoustic reconstruction for photothermal imaging." Bioengineering, Vol. 5, No.3: p 1-9, 2018.

[5] G. Thummerer, G. Mayr, M. Haltmeier and P. Burgholzer. "Photoacoustic Reconstruction from Photothermal Measurements including Prior Information." Photoacoustics-submitted.

[6] G. Mayr, G. Stockner, H. Plasser, G. Hendorfer and P. Burgholzer. "Parameter estimation from pulsed thermography data using the virtual wave concept". NDT and E International, Vol. 100, p. 101-107, 2018.

[7] P. Burgholzer, J. Bauer-Marschallinger, H. Grün, M. Haltmeier and G. Paltauf. "Temporal back projection algorithms for photoacoustic tomography with integrating line detectors." Inverse Problems, Vol. 23, No.6: p 6580, 2007.

[8] N. Parikh and S. Boyd, "Proximal Algorithms," Found. Trends Optim. 1, 127-239, 2014.

[9] F. J. A. Artacho, J. M. Borwein and M. K. Tam, "Recent Results on Douglas-Rachford Methods for Combinatorial Optimization Problems," J. of Optim. Theory and Applic. 163, 1-30, 2014.

[10] R. Bouldin, The pseudo-inverse of a product, SIAM Journal on Applied Mathematics, vol .24, no. 4, pp. 489-495, 1973. 